\title{
Las revistas impresas 'Educación' y la (re)orientación de las prácticas pedagógicas. Historias vinculadas entre São Paulo y Bogotá, 1932-1939 ${ }^{1}$
}

\author{
Carlos Jilmar Díaz Soler ${ }^{2}$ \\ Universidad Distrital Francisco José de Caldas (Colombia) \\ cjdiaz@etb.net.co
}

Recepción: 27/06/2012

Evaluación: 01/10/2012

Aceptación: 03/11/2012

Artículo de Reflexión

DOI: http://dx.doi.org/ 10.9757/Rhela.20.05

\section{RESUMEN}

Este artículo se inscribe en un marco investigativo que busca comprender los dispositivos de producción y de circulación de ideas. Para ello, asume investigativamente dos revistas destinadas a la formación de profesores. Tituladas Educação y Educación y divulgadas entre los años 1932 y 1939, estas Revistas circularon en las redes públicas de enseñanza de São Paulo (Brasil) y de Bogotá (Colombia), período en el cual, además de ser mantenidas por los respectivos Estados y ser organizadas por grupos articulados a políticas educacionales, apostaron a la (re)organización de las prácticas pedagógicas, puntualmente y, de la cultura, en términos generales, mediante la divulgación de específicos contenidos.

Palabras clave: Revista historia de la Educación Latinoamericana, campo de producción, campo de circulación de ideas en pedagogía, revistas impresas, prácticas pedagógicas, ciencias humanas, politica cultural Brasil-Colombia.

1 Este trabajo presenta materiales de la investigación que se llevó a cabo para la Universidad Estadual de Campinas, UNICAMP, Facultad de Educação, S.P., Brasil. Agradezco a mi orientadora, la profesora Dra. Vera Lucia Sabongi de Rossi. Así mismo, la perspectiva analítica propuesta y el énfasis colocado en el análisis de fuentes documentales está posibilitando el desarrollo de la investigación "Educación Superior, saberes e investigación. El caso del Proyecto Curricular de Ciencias Sociales en la Universidad Distrital Francisco José de Caldas. 1970-2000", desarrollado para el Centro de Investigaciones -CIDC- y el Instituto de Estudios e Investigaciones Educativas -IEIE-, de la Universidad Distrital Francisco José de Caldas.

2 Profesor e Investigador de la Universidad Distrital Francisco José de Caldas. Doctor en Educación, área: Conocimiento, Lenguaje y Arte, por la Universidad Estatal de Campinas, UNICAMP, São Paulo, Brasil. 
The printed magazines 'Education' and the (re) work orientation of the pedagogical practices. Histories linked between São Paulo y Bogotá, 1932-1939

\section{ABSTRACT}

This article is part of a research is framed under a wider research aspect that seeks for the understanding of the production and circulation systems of pedagogic ideas, it takes as an essential part for it two magazines destined for the teachers' development. Both entitled de Educação and Education, and published to the public teaching networks of São Paulo (Brazil) and Bogotá (Colombia), between the years 1932 and 1939, during this period these were supported by the respective States and they are organized by jointed groups to educational policies articulated, and they were in a process of (re)organization of teaching practices, and in the culture in general aspects, through the spread of its contents.

Key words: History of Latin American Education Journal, field of production, circulation of pedagogical ideas, printed magazines, teaching practices, social sciences, political cultural-Brazil-Colombia.
As revistas impressas 'Educação' e a (re) orientação das práticas pedagógicas. Histórias vinculadas entre São Paulo e Bogotá, 1932-1939

\section{RESUMO}

Este artigo se insere em um marco de pesquisa que busca compreender os dispositivos de produção e de circulação de ideias. Para isso, assume pesquisar duas revistas destinadas à formação de professores. Intituladas Educação y Educación e divulgadas entre os anos de 1932 e 1939, essas revistas circularam nas redes públicas de ensino de São Paulo (Brasil)e Bogotá (Colômbia), período no qual, apesar de serem mantidas por seus respectivos Estados e serem organizadas por grupos articulados a políticas educacionais, apostaram a (re)organização das práticas pedagógicas, pontualmente e, da cultura, em termos gerais, mediante a divulgação de conteúdos específicos.

Palavras-chave: Revista história da Educação Latino-americana, campo de produção, campo de circulação de ideias em pedagogia, revistas impressas, práticas pedagógicas, ciências humanas, política cultural Brasil-Colômbia

\section{INTRODUCCIÓN}

Pensar la escuela, las prácticas que la constituyen y la particular lógica con la cual sus agentes actúan, configura en la actualidad un escenario importante para la reflexión en el campo de la investigación en pedagogía y educación. Abrir la caja negra y vislumbrar los dispositivos que constituyen la escuela y que contribuyen a su delimitación y organización pone en escena preguntas relacionadas con la cultura, como categoría para el análisis. Asuntos relacionados con la formación de profesores y la profesión docente, con la infancia y su escolarización, con el currículo y las características que asumen los saberes a enseñar, con las prácticas de lectura y escritura y las representaciones del mundo, son apenas indicios de intereses investigativos que están contribuyendo a reorientar, desde la historia de la educación y la pedagogía, este campo de investigación. 
Atravesada fuertemente por las inquietudes del presente, desde la historia de la educación y la pedagogía se postulan esquemas analíticos que posibilitan vislumbrar la articulación entre, por un lado, los dispositivos generados por la imbricación saber-poder y, por otro, las prácticas que tienen lugar en el cotidiano de la escuela. Para ello, discutir la perspectiva sobre el discurso, el valor de las fuentes documentales y de las herramientas conceptuales pasa a ser imperativo para una historiografía de la educación que se pregunta, entre otros, por el 'objeto' escuela, por el modelo escolar imperante en educación, por la infancia y su escolarización, en pocas palabras, por la construcción histórica que permite comprender la "modernidad como sociedad de la escolarización", ${ }^{3}$ particularizando, en la discusión de esta construcción histórica, los elementos constituyentes de un esquema analítico que posibilite la discusión de la forma que adquiere el modelo pedagógico imperante, así como de los efectos en las prácticas pedagógicas.

Al pasar a ser discutida como efecto de particulares procesos históricos, la escuela es concebida en el marco de la interacción y la disputa, en donde particulares dispositivos pugnan por la imposición y la normalización de un sentido. En esta perspectiva, categorías analíticas como cultura, sujetos y prácticas son consideradas centrales en la discusión sobre la pedagogía y el currículo, siendo debatidas, también, en procesos tendientes a la formación de investigadores.

En esta perspectiva, el énfasis colocado en los soportes materiales obliga también a colocar especial atención en las formas de razonamiento que vehiculizan y que contribuyen a delimitar, a fundamentar, e incluso a legitimar o desacreditar ciertas prácticas pedagógicas, asunto que adquiere inusitada relevancia en la actualidad. Libros didácticos, manuales escolares, impresos y colecciones destinados a los profesores, reglamentos, cuadernos de los escolares, guías curriculares, libros, traducciones, programas de formación, así como estructuras curriculares, por mencionar solo algunos, pasan a ser objeto de un renovado interés investigativo. Como documentos producidos en determinada cultura, en la materialidad de estos 'objetos' quedaron contenidas ciertas formas de razonar, que contribuyeron a orientar y reorientar usos puntuales en la escuela del tiempo, del espacio,

3 Carvalho, (1997), p. 95. 
de la autoridad y de los saberes, por ejemplo, y que, al ser convertidos en documentos relevantes para la investigación, posibilitan re-pensar el cotidiano escolar hoy.

En este marco, privilegiar, como fuente para la investigación en historia de la educación y la pedagogía, las revistas periódicas impresas destinadas al magisterio posibilita acercarse a un testimonio vivo en donde fueron debatidos métodos y concepciones pedagógicas que en su momento un grupo político-pedagógico abanderó. Así, la inusitada importancia que cobró, durante la primera mitad del siglo XX, la organización y puesta en circulación de revistas impresas destinadas al magisterio posibilita acercarse a preocupaciones y disputas que en su momento fueron relevantes.

Tanto en Brasil como en Colombia se evidencia en las primeras décadas del siglo XX una creciente actividad editorial: dinámica donde el bullir de las ideas circula en diferentes formatos. Así, desde distintos flancos, mediante la palabra escrita, se difunden idearios políticos, cuestionamientos a la forma en que funciona la sociedad, así como novedades científicas y asuntos que generaron debate en el periodo analizado. Las revistas impresas destinadas al magisterio se convirtieron en un destacado instrumento, en la tentativa de hacer circular a un número mayor de profesores perspectivas sobre el mundo, la cultura y la escuela, acorde con los intereses de sus editores.

El análisis de estas revistas posibilita aprehender la particular lógica de funcionamiento del campo educativo. En las revistas destinadas al magisterio es posible encontrar, gracias a su particular organización, elementos para comprender, no solo el proceso de organización de los nacientes sistemas de educación en América Latina, sino también los debates que contribuyeron a la instauración de la perspectiva pedagógica que hoy se acarrea. En las páginas de estas revistas se encuentran indicaciones sobre cómo se concibió la práctica pedagógica, cómo debería ser y, sobre todo, elementos para su cuestionamiento. Contienen, también, en el periodo de este análisis, disputas específicas que dan cuenta del particular momento en el cual se incorporan las nacientes ciencias humanas al debate pedagógico, lo que contribuyó, como como se verá, a su reorientación. Así mismo, estas revistas presentan al público reivindicaciones magisteriales y una amplia bibliografía. Gracias a la organización de estas revistas impresas se cuenta hoy con materiales para investigar la memoria de la educación y la pedagogía y, mediante su análisis, 
es posible pensar y repensar las especificidades y los elementos comunes para una historia de la educación y la pedagogía en el continente.

Específicamente, el análisis de las revistas 'Educación' permite vislumbrar el ejercicio del poder, en la manera en que fueron seleccionados, organizados y presentados diversos conocimientos a profesores de enseñanza primaria en dos contextos político-culturales. Estas revistas pedagógicas son, a la vez, una conjunción entre los procesos económico-políticos y los conflictos en el saber. $\mathrm{O}$, en otras palabras, como una de las modalidades del ejercicio de poder, las revistas pedagógicas son un medio que posibilitó presentar unos conocimientos sobre el mundo que, a su vez, fueron utilizados como instrumentos para actuar sobre el mismo mundo. Es posible, entonces, pensar estas revistas como un medio a través del cual hicieron circular propuestas acerca de la pedagogía y de la sociedad.

Como revistas impresas, organizadas, materializadas y destinadas al magisterio, con el objeto de difundir específicas formas de razonamiento, con el propósito de debatir y dar respuestas a problemas puntuales, las revistas 'Educación' son comprendidas como vehículos utilizados para divulgar el ideario político-pedagógico de grupos empeñados en la discusión sobre la estructuración del campo educativo, en la tentativa de tornar hegemónicos tales conocimientos. ${ }^{4}$ Como producto de una civilización que ha privilegiado y legitimado la palabra escrita, estas dos revistas posibilitan un análisis de caso. ${ }^{5}$ Como corpus documental, estas dos revistas, destinadas a la lectura del magisterio, posibilitan comprender el marco de actuación propuesto a los profesores; es decir, propuestas de formas de razonamiento desde donde deberían actuar pedagógicamente.

Entonces, asumirlas como corpus documental para el análisis implica hacer dos precisiones. La primera permite de entrada proponer una diferenciación entre corpus y objeto(s) de investigación, pues pertenecen a distintos niveles de análisis: el primero, el corpus, de cada una de las revistas impresas, pertenece al mundo sensible; el segundo, el objeto de investigación, es inteligible;

4 Antonio Gramsci (1891-1937), propone comprender la hegemonía como un complejo entramado entre familias, iglesias, sindicatos, clubes, redes institucionales, que contribuyen a unificar el horizonte institucional y moral de una sociedad. Orientación que, poco a poco, conquista el sentido común.

5 La noción de caso, si bien remite al análisis individual, busca, también, una manera de particularizar el fenómeno colectivo de la proliferación de revistas que, desde el siglo anterior y de manera similar en estos dos contextos, se estaba produciendo. Es una manera de identificar las singularidades del fenómeno político de los usos de la palabra escrita, queriendo integrarlo en un marco de reflexión más amplio. 
nos encontramos, en esta segunda diferenciación, con una amplia gama de 'objetos' que requieren procesos de inteligibilidad: desarrollo, inteligencia, aptitud, infancia, escuela, por ejemplo.

La segunda precisión, que atañe también a los contenidos, está relacionada con que dichos contenidos, que circularon en las revistas destinadas al magisterio, fueron producidos en otros escenarios; asunto que posibilita establecer diferencias entre, por un lado, un campo de producción simbólica -como el de la ciencia-y un campo de recontextualización-como el brindado por las maneras como en cada contexto político circulan y se asumen dichas elaboraciones y que quedaron consignadas en las discusiones que cada una de las revistas propone-. Las diferencias, que justifican la introducción del concepto de recontextualziación -propuesto por B. Bernstein- están en el hecho de que los intelectuales, interesados en la discusión político pedagógica, o los profesores que escribieron en cada una de estas revistas no pertenecen a un campo precisamente donde se produzca el saber, buscan, mediante la agitación de algunas ideas, proponer ciertas transformaciones de las prácticas de las cuales hablan; las elaboraciones contenidas en las revistas están insertas en un campo donde se lleva lo producido en ciertos campos de producción (no todos), en función del contexto al que se destinan, el cual, desde asuntos distintos a los propios del campo de producción, está tensionado.

Se encuentra entonces que algunos elementos propuestos por Bourdieu, con su teoría de campo, contribuyen también al análisis propuesto sobre estas dos revisas. Perspectiva que posibilita condiciones para diferenciar entre los componentes del corpus y las especificidades inteligibles por la teoría.

\section{El corpus documental}

Como centro de gravedad, entonces, para la descripción y el análisis de esas formas de razonamiento se seleccionan dos revistas dirigidas a los maestros. Revistas que circularon hacia la década del treinta del siglo XX. Intituladas 'Educación', impulsadas por sendos grupos políticos y con apoyo financiero de cada uno de los Estados, estas dos revistas circularon en las ciudades de São Paulo y Bogotá, respectivamente. El acotamiento temporal -1932-1939para esta investigación se circunscribe al periodo en el cual 'Educación', la revista de São Paulo, está bajo la dirección del Departamento de Educación 
del Estado. Acotamiento en el cual se inscribe, también, el periodo de edición de 'Educación', en Bogotá-1933-1935-.

El análisis de estas dos revistas permite entrever las ideas con las cuales políticos y pedagogos, tanto para Brasil como para Colombia, enfrentaron dos complejos problemas: estructurar un moderno sistema educativo y escolarizar masivamente a la infancia pobre.

Para el caso de Educação, la revista “Órgano del Departamento de Educación del Estado de São Paulo", el grupo político-pedagógico que asumió su edición, desde enero de 1932, fue la Sociedad de Educación, grupo conformado por connotados intelectuales inquietos con la cuestión educativa. Para el periodo de nuestro interés, este grupo toma el control de Educação y difunde en esta revista su propuesta pedagógica.

En el marco de la reconfiguración de la Revista, el volumen I, de marzo de 1932, presenta con júbilo la designación de Fernando de Azevedo como Director General de Instrucción Pública del Estado de São Paulo en 1932 y registra también los asistentes al evento. ${ }^{6}$ Así mismo, en este número se recogen las palabras que pronunció Fernando de Azevedo al asumir como nuevo Director General de Instrucción Pública del Estado de São Paulo.

Si ha alguém a que se devia dispensar o discurso tradicional na solenidade de investidura neste cargo, seria o homem a quem tudo se poderá negar menos a fidelidade ás suas condições, o ardor e a pertinácia no defendê-las e o impeto em transformá-las em realidade, em passar das idéias aos fatos, pensamento á ação. Na longa campanha de renovação educacional em que tenho tomado grande parte, disputando a meus eminentes companheiros de idéias a primazia no esforço e na tenacidade, através de obstáculos de toda natureza, fui obrigado, já uma vez no Distrito Federal, a trocar o meu lugar na trincheira, na defesa das idéias, por um posto de direção, em que se me abria oportunidade de construir e de submeter ás provas mais duras o meu empenho em realizá-las.?

6 Nace en São Gonçalo do Sapucaí, MG, el 2 de abril de 1894, y muere en São Paulo, SP, el 18 de septiembre de 1974. Abogado y profesor, se integra al movimiento reformador en la década del 20, impulsado por la Associção Brasilera de Educação, fundada en 1924. Su carrera en el magisterio se inicia en 1914 en el Ginásio Do Estado en Belo Horizonte, como profesor de Latin y Psicología. Además fue Director de la Facultad de Filosofía, Ciencias y Letras de São Paulo (1941-42); Miembro del Consejo Universitario por más de doce años, desde la fundación de la Universidad de São Paulo; fue, también, Secretario de Educación y Salud del Estado de São Paulo (1947); Director del Centro Regional de "Pesquisas Educacionais", que él instaló y organizó (1956-61); Secretario de Educación y Cultura en gobierno del prefecto Prestes Maia (1961); redactor y crítico literario de "O Estado" de São Paulo (1923-26), periódico en que organizó y dirigió, en 1926, de los interrogatorios, uno sobre la arquitectura colonial, y otro sobre Educación Pública en São Paulo, abordando los problemas fundamentales de la enseñanza de todos los grados y tipos, e iniciando una campaña por una nueva política de educación y por la creación de universidades en Brasil.

7 Discurso do Dr. Fernando Azevedo, Educação, Vol.: I (1933): 195. 
Con estas palabras Fernando de Azevedo se refiere a su cargo como director de la Instrucción Pública en el Distrito Federal entre 1927 y 1930, en donde promovió una amplia reforma. Así mismo, tácitamente hace referencia a que en 1931 funda la Biblioteca Pedagógica Brasilera en la Compañía Editora Nacional. Y que, junto con otros 25 educadores e intelectuales, suscribió el Manifesto dos Pioneros da Educação Nova. Manifiesto que, precisamente, fue publicado en la Revista Educação, en el volumen I, en 1932.

Con respecto a las ideas, Fernando de Azevedo estaba consciente de que él representaba un grupo que disputaba una perspectiva, una manera de comprender la educación, puntualizando que "trocar o meu lugar na trincheira" era continuar en la disputa, ahora desde otro lugar, frente a grupos políticos enfrascados en la re-orientación de la educación. Así mismo, sabía que tener la posibilidad de estar en cargos directivos era propicio para materializar las ideas de su grupo político-pedagógico.

En el debate educacional de los años treinta en Brasil, varios grupos pedagógicos disputaban el control político del aparato educativo. Desde los años veinte, en la Asociación Brasilera de Educación (ABE), principal instancia de articulación del llamado Movimiento de Renovación Educacional, se congregó un grupo de intelectuales en torno a lo que se denominó la causa "cívico-educacional". En la coyuntura del crecimiento del aparato estatal y la disputa por la hegemonía política, así como en la lucha por el control del aparato escolar, estos educadores antagonizan cuando se tornó central la disputa por el ideario pedagógico. En esta disputa dos grupos se constituyen, rivalizando mediante sendas propuestas político-pedagógicas por el control conceptual y técnico de las escuelas: los 'católicos' y los 'pioneros'. ${ }^{8}$

As nomeações designam, no caso do primeiro grupo, setores militantes do laicato intelectual católico que haviam integrado a $A B E$ nos anos vinte e que abandonam em 1932, passando a se articular na Associação dos Professores Católicos do Distrito Federal e no Centro D. Vital de São Paulo, inicialmente, e, a partir de 1934, na Confederação Católica Brasileira de Educação. No caso do segundo, o nome designa alguns signatários do Manifesto dos Pioneiros da Educação Nova que -tendo participado em Conferencias Nacionais de Educação promovidas pela $A B E$ e ocupado postos governamentais, na qualidade de "técnicos"

8 Chagas de Carvalho, Marta Maria. Molde nacional y fôrma cívica, (1998): 133-185. 
empenhados na reforma dos sistemas estaduais de ensino, assumiram o controle da Associação Brasileira de Educação a partir de 1932. ${ }^{9}$

En este marco, de la mano de la nueva dirección y con la directriz señalada en el manifiesto, la revista Educação se convirtió en el portavoz del grupo político-pedagógico de los llamados 'Pioneros'. Los conocimientos que circularon fueron proclamados por estos intelectuales como legítimos, disputándole, con ello, el espacio simbólico a otros grupos político-pedagógicos establecidos. Para efectos de esta investigación, que toma como corpus documental las páginas de Educação, se hará una aproximación a la propuesta pedagógica de este grupo. El Grupo de intelectuales antagónico, aglutinado en torno a la Confedereção Católica Brasilera de Educação, reunidos en torno al Centro D. Vital de São Paulo, organizó, también, una revista: A ordem, cuya pretensión fue trabajar en la dirección de un movimiento de cristianización de las élites y, a través de ellas, la cristianización del pueblo, del Estado, asuntos que, consideraban, debían quedar consignados en la legislación. ${ }^{10}$

El recorte temporal, como ya fue señalado, propuesto para esta pesquisa toma como referente inicial el año de 1932. Año en el cual la revista Educação, pasa a ser dirigida por la Dirección General del Estado de São Paulo. En el primer número de esta nueva era, marcado con el volumen VI y que circuló entre enero, febrero y marzo de 1932 con el número 1, aparece un texto titulado A reconstrucção educacional no Brasil. Ao povo e ao governo, firmado por Fernando de Azevedo, Antônio de Sampaio Doria, ${ }^{11}$ Anisio Spinola Texeira, ${ }^{12}$ Manoel Bergstrom Lourenço Filho, ${ }^{13}$ Afranio Peixoto, Roquete

9 Chagas de Carvalho, Marta Maria. "O território do consenso e a demarcação do perigo: política e memória no debate educacional dos anos 30", (2002): 14 .

10 Nunes, Clarice. "O Estado novo e o debate educacional nos anos 30", (2002), 29.

11 Nace en Belo Monte, provincia de Alagoas en marzo de 1883 y muere en 1964. Abogado de formación, fue profesor de la disciplina de psicología y lógica en el Gimnasio Macedo Soares. Periodista y redactor jefe de O Imperial, en Rio de Janeiro. En 1914, en São Paulo, es nombrado profesor catedrático de la cátedra de Psicología, Pedagogía y Educación cívica de la Escuela Normal Secundaria de São Paulo. Autor de varios textos Principios de pedagogía, 1914. Ensaios, 1915. Psicología e educação. Como se ensina e Como se aprende a lengua, 1923. Fue director de instrucción pública responsable por una controvertida reforma de enseñanza en São Paulo emprendida por el Decreto 1750 de 8 de diciembre de 1920 y revocada en 1925. Desde esta reforma trató de encontrar una estrategia de generalización de la escuela proponiendo la reducción de la obligatoriedad escolar para dos años. El analfabetismo alcanzó el estatuto de "cuestión nacional por excelencia" y, en consecuencia, priorizó la extensión de la escuela a las poblaciones hasta entonces marginalizadas. Ver Spedo Hilsdorf, Jorge Warde, Chagas de Carvalho. "Apontamentos sobre a história da escola e do sistema escolar no Brasil" 2004, 162-164.

12 Nace en Bahia en julio de 1900 y muere en Rio de Janeiro en marzo de 1971. Abogado de la Universidad de Rio de Janeiro y Master en artes por el Theaters College da Columbia University. Se desempeñó como Inspector General de Enseñanza en Bahia. Entre los años 1924 a 1929 realiza la reforma de la instrucción pública en el Distrito Federal. Director de Instrucción Pública de Rio de Janeiro, entonces llamada Distrito Federal, entre 1931 y 1935. Lector crítico de John Dewey (1859-1952) traduce al portugués varias de sus obras.

13 Nace en marzo de 1897 en São Paulo y muere en Rio de Janeiro en 1970. Para él el problema de la educación nacional se confundía con el de la organización social, siendo la educación entendida como instrumento de muchas técnicas que deberían ser armonizadas para que se pudiesen alcanzar los fines deseados. Se dedicó a dos áreas de conocimiento prioritariamente, la psicología y la estadística, que, exponía, convergían conceptualmente en el aparato teórico y en las técnicas necesarias a la tarea 
Pinto, Mario Casasanta, J. G. Frota Pessoa, Julio de Mesquita Filho, Raul Briquet, C. Delgado de Carvalho, J. P. Fontenelle, A. Ferreira de Almeida Junior, Roldão Lopes de Barros, Noemy M. Da Silveira, Hermes Lima, Attilio Vivacqua, Francisco Venancio Filho, Paulo Maranhão, Cecilia Meirelles, Edgar Sussekind de Mendoça, Armando Alvaro Alberto, Garcia de Rezente y Nobrega da Cunha. Firmantes de los cuales se presentan rasgos de su actividad pedagógica y política solo de aquellos con mayor actividad en la Revista de que acá se analiza.

Este documento, conocido también como Manifiesto de los pioneros por la nueva educación, al ser el que inaugura una nueva época de la Revista, señala también, la directriz que este grupo político, ahora en el poder, buscaba darle a la Dirección General de Educación y, por este medio, a los procesos educativos en la ciudad de São Paulo. ${ }^{14}$ Estos ideales contribuyeron también, para este periodo, a la orientación ideológica de la Revista. Llama la atención que los firmantes de este primer artículo pertenecen a la Sociedad de Educación de São Paulo. ${ }^{15}$

Por su lado, en Bogotá, la revista Educación, Órgano de la Facultad de Educación, adscrita a la Universidad Nacional en 1933, organizada por un grupo de intelectuales vinculados al gobierno, se convirtió, también, en difusora de ideales pedagógicos. La creación formal de la nueva Facultad de Ciencias de la Educación, mediante el decreto 1990 de 1933, procedió a establecer lazos estrechos entre el Estado y la universidad, en la tarea de definir los saberes necesarios para el ejercicio del magisterio, disputándole con esto

de la educación y la organización social. La psicología aplicada, además de proporcionar conocimientos sobre los educandos, sería también instrumento de análisis de las profesiones, estudiaría las cuestiones relativas a la selección profesional y la adecuación del trabajo a las condiciones generales del comportamiento del trabajador. La estadística y la psicología contribuirían teóricamente y mediante técnicas a la tarea de la educación y de la organización social, que pasa a ser entendida en un cuadro más amplio de manipulación técnica de la sociedad. En 1935 fue nombrado profesor de psicología educativa de UDF. En 1937 fue nombrado miembro de Conselho Nacional de Educação. En 1940 es nombrado Director del Instituto Nacional de Estudios Pedagógicos. En 1962 participa en la elaboración de la ley de reglamentación de la profesión de psicólogo.

14 Este manifiesto se convirtió en una propuesta de intervención de las elites y radicalizó el escenario político del periodo ya que los pioneros se proponían una intervención racional en el sistema educacional y proponiendo una "educación científica del espíritu". Por su lado intelectuales ligados a la jerarquía católica condenaron duramente el contenido del manifiesto y a sus firmantes. Críticas que se condensaron y fueron puestas en circulación en la Revista A Ordem, del Centro Dom Vital. Ver: Xavier, Maria do Carmo (Org.). Manifesto dos pioneiros da educação. Um legado educacional em debate, 2004:21.

15 La lucha ideológica por el control de aparato político de la educación en Brasil, comenzando la década del treinta, es intensa. La Revista Educación se convierte en el órgano difusor del Grupo que organizó el Manifiesto, en el cual, además de los ya señalados estaba Gustavo Capanema, quien sucedió a Francisco Campo en el Ministerio de Educación y Salud. Francisco Campo, protagonista de primer orden en la discusión educativa desde la década del veinte, representaba los intereses de la iglesia católica en el poder, el otro gran protagonista de esta lucha ideológica por el control del aparato educativo. La revista A Ordem fue su medio privilegiado de difusión de contenidos y de oposición al régimen político implantado desde 1930. Ver, [Schwartzman, Simon, Helena Maria Busquet Bomeny, Vanda Maria Ribeiro Costa. Tempos de Capanema,1984:61-66]. Para un análisis de este Documento - Monumento que fue titulado "Manifiesto de los Pioneros" ver [Xavier, Maria do Carmo (Org.). Manifesto dos pioneiros da educação. Um legado educacional em debate]. 
a la iglesia católica el establecido monopolio de la educación. La Facultad de Ciencias de la Educación aparece en un momento de transición de la Universidad Nacional de Colombia, caracterizada por una reforma que buscaba mayor autonomía, tanto del Ministerio de Educación, como mayor articulación entre sus Facultades, además de la construcción de un campus propio, que evitase su dispersión en la cuidad. ${ }^{16}$ Estratégicamente, con la oficialización de la nueva Facultad de Ciencias de la Educación, fueron legitimados discursos que contribuirían al proceso formativo del magisterio, lo cual posibilitaría su posterior profesionalización. La creación de la Facultad de Ciencias de la Educación constituye uno de los aspectos, señalados desde la historiografía nacional, como central para la discusión pedagógica. Fue corta la vida de la Facultad de Ciencias de la Educación en la Universidad Nacional, ya que en 1936 fue transformada en Escuela Normal Superior ${ }^{17}$.

Agustín Nieto Caballero, el 15 de abril de 1932, anunció la apertura de la Facultad de Ciencias de la Educación en la Universidad Nacional. Aspiraba que por medio de esta Facultad el Estado definiera su concepto y las normas aconsejables, tanto para la escuela primaria, como para la complementaria, así como para las instituciones universitarias. Este anuncio proferido por el Inspector General de Educación Nacional señala una nueva política educativa encaminada a establecer nuevos conocimientos para el ejercicio del magisterio, legitimados desde los saberes científicos y mediante una institución encargada de su difusión: la universidad. Con ello se decide, desde el Estado, modernizar y profesionalizar la instrucción, mediante la formación de profesores para las escuelas. El 5 de diciembre de 1933 el entonces presidente de Colombia Enrique Olaya Herrera expide el decreto que creaba formalmente la Facultad de Ciencias de la Educación en la Universidad Nacional de Colombia. Con un énfasis marcado por la circulación selectiva de diferentes

$16 \mathrm{Su}$ antecedente se remonta al año de 1830, cuando comenzó a funcionar con profesores y estudiantes que mantenían viva la experiencia de la Expedición Botánica la Universidad Central de la República (con sedes en Bogotá, Caracas y Quito) como primera expresión jurídico-institucional de la Universidad pública en Colombia. Luego los gobiernos federalistas y radicales bajo el argumento de que las universidades eran monopolios de enseñanza y de trabajo que atentaban contra la libertad individual, cierran la universidad Central hasta que en 1864, José María Samper, antiguo radical, presentó un proyecto de ley al Congreso, de lo que llamó Universidad Nacional de los Estados Unidos de Colombia. El 22 de septiembre de 1867, mediante la Ley 66 expedida por el Congreso es oficialmente fundada como tal la Universidad Nacional de Colombia. Entre 1903 y 1940 se crearon en la Universidad más de 20 carreras, entre las que se encuentran: Arquitectura, Enfermería, Farmacia, Ingeniería Química, Medicina Veterinaria, Odontología y Química. A finales de la década de los 60 se le dio impulso a los programas de maestría en la Universidad Nacional y en país. Los primeros programas, a nivel de maestría fueron creados entre 1967 y 1973 y en 1986 abrieron sus puertas los primeros programas doctorales del país en áreas como la física y Matemáticas.

17 Para un análisis de la conformación y transformación de ésta institución ver: Martha Cecilia Herrera y Carlos Low, Los intelectuales y el despertar cultural del siglo. El caso de la Escuela Normal Superior. Una historia reciente y olvidada. Universidad Pedagógica Nacional, 1994. 
discusiones pedagógicas, dentro de los cuales se destacan, entre otros, John Dewey, Ovidio Decroly, E. Claparède, Alfred Binet, se procedió, desde esta recién creada Facultad de Ciencias de la Educación, al establecimiento de los Programas de Ensayo para la Escuela Primaria en 1935.

A partir de 1930, y ante la decidida oposición del Partido Conservador y de la Iglesia, los gobiernos liberales intensificaron los esfuerzos por centralizar y unificar la educación pública, para dotarla de un carácter nacional que superara la atomización resultado de la autonomía de la que gozaban los departamentos y las libertades que los gobiernos conservadores le habían concedido a las instituciones católicas privadas. Los principales elementos del proceso de centralización fueron: a) el establecimiento de la Inspección Nacional de educación Primaria y Normalista, en 1931; b) el relevo gradual de los inspectores provinciales y locales por los directores departamentales de educación y los rectores de las normales, ligados al Estado central; c) la progresiva intervención de la inspección central sobre la educación privada; d) la unificación nacional de los programas de primaria, secundaria y educación normalista entre 1932 y 1935; el inicio de la nacionalización de los colegios municipales y departamentales en 1938; el incremento de la financiación del Estado central, a partir de la Ley 30 de $1944 .{ }^{18}$ En el marco de un debate entre diferentes concepciones pedagógicas que buscaban definir la función y el sentido de la educación, se instauró, en esta naciente Facultad, una fuerte disputa por la manifiesta intención del Estado de apropiarse del monopolio de los saberes necesarios para el ejercicio magisterial. Este ejercicio político de institucionalización universitaria de unos saberes que contribuirían a circunscribir las prácticas pedagógicas, se caracterizó por la prioridad que tuvieron los discursos científicos sobre lo social, en los que cabe mencionar la sociología y la moderna psicología.

En este marco de tensiones políticas se creó la revista Educación, órgano de difusión de la Facultad de Ciencias de la Educación. La puesta en marcha de Educación buscó, en palabras aparecidas en el primer número de la Revista y escritas por Rafael Bernal Jiménez: "fijar nuestro concepto

18 Quiceno, Humberto, Javier Sáenz Obregón y Luis Arturo Vahos. “La instrucción y la educación pública en Colombia: 1903-1997”, (2004), 141. 
espiritualista de la cultura, así como determinar nuestra posición en frente de los problemas educativos colombianos". ${ }^{19}$

Desde luego esta Revista ha de ser el órgano autorizado de la Facultad Nacional de Educación; con lo cual no se quiere decir que deba limitarse a ser el monótono registro de la tarea escolástica, sino la voz de un centro de actividad y de estudio de nuestros problemas educativos y a reflejar este criterio sobre la actividad nacional. Por otra parte esta Revista abriga el propósito de contribuir a la mejor información de profesores y maestros, teniéndolos al corriente de las principales iniciativas que vayan surgiendo en el campo de la metodología particular. ${ }^{20}$

Esta Revista vio su primer número en el mes de agosto de 1933. Sus destinatarios fueron los maestros de enseñanza primaria y secundaria, así como los inspectores escolares del gobierno. La Revista fue catalogada como el órgano autorizado de la Facultad Nacional de Educación, siendo la voz del centro académico y asumiendo para su debate, como problema político, la educación nacional. El propio Rafael Bernal Jiménez (Director de la revista) expresó que el objetivo de la Revista era servir a los intereses intelectuales y materiales del magisterio, que pretendía organizar como "entidad respetable, para tomar la injerencia que le corresponde en la orientación del pensamiento colombiano y en la preparación de los destinos de la patria". El objeto de la Revista fue, entonces, contribuir al desarrollo de la educación en Colombia, servir de tribuna a los propagadores de las nuevas ideas pedagógicas y facilitar el flujo de información sobre las experiencias que estaban en curso, obrando como mediador entre el Ministerio de Educación y los maestros. ${ }^{21}$

La Revista contó con veintinueve (29) números y cada número iniciaba siempre con un escrito elaborado por el Director Rafael Bernal Jiménez, ${ }^{22}$ que promovía el punto de vista sobre el contexto político y educativo. Cada número de la Revista finalizaba con escritos que relacionaban crónicas universitarias, reflexiones sobre la formación del magisterio, actividades de las

19 Bernal Jiménez, Rafael, Educación Vol.: 1, (1933): 1- 4.

20 Bernal Jiménez, Vol.: 1, (1933): 4.

21 Bernal Jiménez, Educación Vol.: 1, (1933): 1- 4.

22 Rafael Bernal Jiménez (1898-1972) nace en Boyacá. Se forma derecho en el Colegio Mayor del Rosario, con especialización en derecho penal de la Universidad de Roma. Entre 1924 y 1928 ocupa el cargo de Director de Instrucción Pública en Boyacá. Entre 1933 y 1935 se desempeñó como Rector de la Facultad de Educación y Director de la Revista Educación en Bogotá, de la cual fue un activo articulista. Entre 1928 y 1932 fue delegado por Colombia en conferencias internacionales en el área de derecho laboral y en congresos de educación. 
Facultades de Educación y noticias del mundo académico: congresos, nuevos conceptos, bibliografía en el ámbito de la pedagogía. Entre los colaboradores destacados se encontraban Agustín Nieto Caballero, ${ }^{23}$ Miguel Jiménez López, ${ }^{24}$ Julius Sieber, ${ }^{25}$ Germán Arciniegas, Félix Restrepo, Juan Manuel Huertas, Luis López de Mesa, ${ }^{26}$ Norberto Solano Lozano, Raymond Buyse y Paulina Gómez, entre otros. Desde los diferentes artículos publicados, se "aspiraba a formar un centro de permanente y noble inquietud en torno a los fundamentales problemas de la educación pública". ${ }^{27}$

Un significativo volumen de artículos en estas dos estas revistas gira en torno a temas relacionados con la infancia, la psicología, la alfabetización, la escuela activa, la medición de la inteligencia y de las aptitudes, la fatiga, la escuela rural, los comedores escolares, la higiene, entre otros. Específicamente para esta investigación fueron seleccionados artículos en los cuales se discutía sobre la estructuración del naciente sistema educativo, la realidad educativa, el sentido de escolarizar a los niños, así como artículos relacionados con la psicología, la inteligencia, las aptitudes, la necesidad de su medición y la utilidad de estos saberes en la práctica pedagógica.

Así, los dos grupos políticos, tanto en São Paulo como en Bogotá, son portadores de nuevos conocimientos sobre la pedagogía y la infancia y, mediante la palabra escrita, contribuyen a difundir específicas formas de razonamiento. La escuela pública, la escuela a la medida, la escolarización de las camadas populares infantiles, son elementos clave de una política del conocimiento que miraba hacia fuera, buscando en los referentes europeos y anglosajones inspiración para pensar las complejas problemáticas que incomodaban a pedagogos y políticos en estos dos contextos. ${ }^{28}$

23 Agustín Nieto Caballero (1889-1975). Hijo de prestigiosos empresarios. En el extranjero estudió su bachillerato y cursó estudios de Derecho, Filosofía, Ciencias de la Educación, Bilogía, en Francia y Psicología, en el The Teacher College de la Universidad de Columbia en Nueva York. En 1913 regresa a Colombia y junto con un grupo de colegas se propuso fundar el Gimnasio Moderno del cual fue su rector hasta 1924. Entre el 1933 y 1935 participa activamente en la Revista Educación. Entre 1932 y 1936 ocupa el cargo de Director de la sección de Educación Primaria y normalista del Ministerio de Educación. Asistió a varios congresos internacionales de educación y presidió la Quinta Conferencia Internacional de Instrucción Pública reunida en Ginebra en 1936. Entre 1938 y 1941 fue rector de la Universidad Nacional. Además fue delegado por Colombia, en varias ocasiones, ante la UNESCO y la OEA.

24 Miguel Jiménez López (1886-1965) médico psiquiatra, tío de Rafael Bernal Jiménez. Fue uno de los que abrió la polémica sobre la degeneración de la raza en 1918. Desde 1925 hasta 1928 fue embajador en Berlín.

25 Pedagogo Alemán (1892-1963). Llegó por primera vez a Colombia en 1926.

26 Luis López de Mesa, nació en Antioquia en 1884 y muere en Medellín en 1967. Obtiene el título de médico de la Universidad Nacional de Colombia en 1912. Fue especialista en psiquiatría de la Universidad de Harvard. Realizó estudios complementarios en Inglaterra, Francia, Alemania e Italia. Además de dictar las cátedras de Psiquiatría, Sociología y Arte en la Universidad Nacional fue su rector en 1948. Fue Ministro de Educación Nacional en el periodo comprendido entre 1934 y 1935. Fue promotor e impulsor de la Campaña de Cultura Aldeana en Colombia entre 1934 y 1936.

27 Bernal Jiménez, Educación Vol: 1, (1933),1- 4.

28 Además de los autores señalados anteriormente, en las páginas de estas dos revistas son profusas las referencias a pedagogos de reconocida trayectoria. Por citar solo algunos: Dewey Jonh, La escuela y la sociedad, Librería española, Madrid (1929). Ferrière, 
Estos gobiernos republicanos de la década del treinta, tanto en Brasil como en Colombia, enfrentaron la compleja problemática de configurar instituciones que posibilitasen, mediante la delimitación y articulación de las instancias apropiadas, la formación de los maestros en la tarea política de instruir a grandes camadas de niños pobres. Tarea política que implicaba la espinosa cuestión de definir y delimitar, tanto las instituciones encargadas de formar a los aspirantes al magisterio, como establecer los conocimientos necesarios que orientarían la práctica pedagógica. Se trataba, entonces, de organizar las instituciones y definir los estamentos, con la pretensión de obtener el monopolio educativo a nombre del Estado. Y, a través de estos, difundir referentes conceptuales desde donde debían actuar los profesores. En este marco, las revistas impresas de circulación periódica se constituyeron en un escenario privilegiado para el proceso de formación de maestros. Las nuevas orientaciones y referencias científicas, legitimadas mediante el ejercicio del poder, circularon, también, mediante la estratégica difusión de revistas impresas destinadas al magisterio.

\section{CONCLUSIÓN}

Una de las preocupaciones que ocupó a pedagogos y políticos es el asunto relacionado con la infancia. Preocupación evidente consignada en las páginas de las dos revistas Educación; siendo la infancia de camadas populares, descrita de manera similar: en "situación de riesgo", para lo cual era indispensable la "atención directa del Estado", la "construcción de un significativo número de escuelas para su protección", así como la formación de maestros para su adecuado cuidado y formación; pero sobre todo, se discutía en estos dos escenarios políticos, la necesidad de formar una "conciencia nacional" alrededor de la importancia de este periodo de la vida, tanto para los individuos, como para la sociedad. ${ }^{29}$

Adolfo. Problemas de educación nueva, Madrid, Francisco Beltrán (1930). Montessori, María. El niño. El secreto de la infancia, México, Editorial Diana (1982).

29 La institucionalización de la escuela obligatoria, en tanto mecanismo de control social, es un dispositivo moderno para la infancia de sectores populares. En sus años de establecimiento, la obligatoriedad solo se aplicó a las "clases bajas", ya que las altas no dudaban en instruir a sus hijos. La escuela se convertía así en la única vía de acceso a la civilización de las camadas populares. Los científicos de lo social, tanto en Brasil como en Colombia, han privilegiado hasta ahora las investigaciones sobre las camadas populares. La manera en que los grupos de élite construyen su manera de ver el mundo y cómo y por medio de qué procesos esos grupos construyen una determinada manera de pensar sobre sí mismos y sobre los otros ha sido motivo de investigación recientemente. Este filón investigativo, desde una perspectiva histórico-sociológica ha interrogado los modos de socialización y de escolarización de los niños y jóvenes oriundos de las familias más privilegiadas. Este filón investigativo comienza a ser explorado. En Brasil (Almeida F., Ana Maria e Alice Nogueira. A escolarizaçao das elites. Um panorama internacional de 
Circuló en estas dos revisas impresas la idea de que desde el discurso de la ciencia se posibilitaría ver la verdadera esencia humana y, por ende, se pensó que se estaba ante la manera como funcionaría la inteligencia y las aptitudes, ideal científico que fue asumido por la pedagogía. En consecuencia, se postuló que la práctica pedagógica debería estar comandada desde los referentes emanados por el discurso científico, escenario desde donde se estaba indicando: qué es un niño, cómo se desarrolla, cómo estimular su interés y las características que debe tener el conocimiento a enseñar, por ejemplo; escenario que, a su vez, permitiría establecer la mejor forma de enseñar y las estrategias necesarias para el adecuado desenvolvimiento de la infancia.

Para la discusión que se realiza, que toma como centro para su análisis las Revistas Educación, impresas en São Paulo y Bogotá, es posible decir que desde el discurso científico se contribuyó a que los saberes soporte para la práctica pedagógica se definieran desde referentes provenientes de la psicología y la sociología, como saberes modernos. Del total de artículos que circulan en estas revistas, los relacionados con estos saberes constituyen un significativo porcentaje, lo cual lleva a pensar que son asumidos como indispensables para actuar sobre los problemas sentidos, en cada uno de estos dos contextos nacionales.

Los métodos de la psicología experimental y aplicada eran el símbolo de la modernidad y la aplicación de los estudios de la psicología del niño y de la inteligencia y, concretamente, del estudio de las aptitudes en los niños, al principio de individualización en la escuela, buscaron tornar científica a la pedagogía en el periodo. De hecho, establecer una la escuela a la medida, como ideal político del periodo era imposible sin un buen conocimiento del alumno y un mejor proceso de orientación escolar. Se asistía, en consecuencia, con esta novedosa preocupación sobre el niño y el énfasis puesto sobre las nacientes disciplinas de lo humano, a la configuración de lo que hoy se denominará el niño escolarizado. Hacer del niño alguien que aprende en la escuela, lo involucró, en consecuencia, de una manera muy particular en las transformaciones

pesquisa, 2002) En Colombia (Prieto, Víctor Manuel. El Gimnasio Moderno y la formación de la Elite Liberal Bogotana: 2000). Finalizando el siglo XIX y durante las primeras décadas del siglo XX la literatura científica vio aparecer un significativo número de trabajos cuyo centro de reflexión fue el niño. Por citar solo algunos ejemplos: Binet Alfred (1909), Las ideas modernas sobre los niños, Fondo de Cultura Económica, México (1985). Claparede E. (1910), Psicología del niño y pedagogía experimental, Madrid. Wallon Henri (1915), La evolución psicológica de niño, Crítica (2000). Marenholtz-Bülow (1896), El niño y su naturaleza, Nueva York, D. Appleton y Compañía Editores. Gaupp Robert (1927), Psicología del niño, Editorial Labor, Barcelona. Dewey, J. (1936), La escuela y el niño, Madrid, Espasa-Calpe S.A. 
del razonamiento sobre lo social, que se asocia con procesos modernizadores. $\mathrm{O}$, en otras palabras, con esta nueva forma de razonar sobre la infancia, que circuló en estas dos revistas, se delimitó con palabras una cierta forma de recortar, de interpretar y de categorizar el mundo social.

Así, privilegiar documentos impresos, de circulación periódica, editados con recursos oficiales, destinados a maestros, en contextos de circulación como São Paulo y Bogotá, configura un particular campo de indagación. En primer lugar, porque para mediados de la década del treinta lo escrito y la ciencia gozan de una inusitada credibilidad política. En segundo lugar, porque había la certeza que, vía procesos de escolarización, las dificultades sociales serían solucionadas. En tercer lugar, por que para este periodo, los asuntos relacionados con la infancia, la psicología y la pedagogía (desarrollo, inteligencia, aptitudes, entre otros) constituyen un predominante campo de investigación, tanto en Europa como en los Estados Unidos. En cuarto lugar, por que los discursos que circulan en estas dos Revistas no logran comprenderse sino por el funcionamiento del conjunto, es decir, estos discursos que quedaron consignados en las páginas de 'Educación', pertenecen, a su vez, a un campo de producción mucho más amplio.

Si los artículos contenidos en estas dos revistas hacen una apelación al carácter verdadero de la ciencia y son presentados como descubrimientos sobre el mundo, es necesario, entonces, asumir algunos elementos propuestos por Bourdieu, con su teoría de campo, para precisar una perspectiva sobre los 'objetos' que estas dos revisas colocaron en circulación. Perspectiva analítica que posibilita diferenciar entre los componentes del corpus y las especificidades inteligibles por la teoría. Lo cual nos coloca en situación privilegiada para analizar asuntos densos relacionados con la pedagogía, el currículo y la práctica pedagógica. Se piensa que esta perspectiva posibilita comprender la circulación social de los enunciados en el campo de la pedagogía, así como asuntos relacionados con la discusión sobre el currículo.

Queriendo precisar, entonces, una diferenciación entre corpus y objeto(s) de investigación, ya que pertenecen a distintos niveles de análisis, es posible decir que el corpus compuesto por cada una de las revistas impresas, pone a disposición de los destinatarios elementos para pensar las características de 
un mundo sensible, mediante la presentación de una perspectiva de ciertos 'objetos': inteligencia, aptitud, infancia, desarrollo, por ejemplo. Gracias al análisis sobre estas fuentes documentales, se percibe que, para el periodo de interés, se estaba ante la creencia que los sujetos están delante de los objetos, que el lenguaje nombra los objetos, que no existen mediaciones. Se postuló que la descripción y manipulación de los 'objetos' (infancia, desarrollo, inteligencia, por ejemplo) correspondía, efectivamente, a la misma manera en la que funcionaba esa 'realidad'. Es decir, se asumía que habría transparencia entre la descripción científica y el objeto de análisis. En consecuencia, el discurso de la ciencia sería el encargado de explicar la 'realidad'.

Lo anterior lleva a avanzar en la asegunda precisión. Es explícito en los contenidos propuestos por las dos revistas que dichos contenidos provienen de ciertos centros de producción académica ubicados en algunas instituciones de Estados Unidos de América (Universidad de Columbia, en Nueva York, por ejemplo) y otros de Europa (Instituto Jean-Jacques Rousseau, en Ginebra, Suiza, por ejemplo). Lo cual posibilita establecer diferencias entre, por un lado, un campo de producción simbólica (como la ciencia) y un campo de recontextualización (como el brindado por las maneras como en cada contexto político circulan y se asumen dichas elaboraciones y que quedaron consignadas en las discusiones que cada una de las revistas propone). Gracias a la introducción del concepto de recontextualización -propuesto por B. Bernstein-, se justifican estas diferencias. En las revistas circulan traducciones y comentarios, por ejemplo, a algunos aspectos de la conceptualización producida desde lo que se puede llamar el campo de producción en la ciencia y que son puestos en función del contexto al que se destinan, tensionado, a su vez, desde asuntos distintos (política) a los propios del campo de producción (lógica interna).

En este marco, la noción de campo, propuesta por Bourdieu y por Bernstein, posibilita presentar un análisis entre discursos y estructuras de conocimiento, lo cual permite vislumbrar la puntual arquitectura con la que opera el campo de producción simbólica, así como la particular lógica del campo de recontextualización -oficial y pedagógico-. La discusión propuesta para el campo de producción simbólica está encaminada a analizar la naturaleza problemática del conocimiento sobre lo social, las formas como buscó su 
legitimidad y los métodos que privilegió para su producción. Destacando que para las llamadas ciencias del hombre, una de sus mayores dificultades reside en el hecho que sus objetos son objeto de luchas o de cosas que se ocultan, que se censuran. Ignorar la estructura que asumió la ciencia en la construcción de los objetos, es decir, la perspectiva desde donde fueron construidos ciertos objetos caros, también, a la pedagogía, implica desconocer para nuestras sociedades la posición ocupada por el campo científico, su lógica y su historia, así como los efectos de una sostenida pedagogización del discurso de las ciencias.

Ahora bien, si para el periodo de interés y con el corpus documental seleccionado, se trató de un discurso con fines pedagógicos, que apeló a la legitimidad provenientes del discurso de ciencia, entonces, se hace necesario avanzar en una discusión sobre el papel pre-figurativo de los procesos de formación de profesores, dado que los procesos de formación de profesores presentan como particularidad la enseñanza de saberes provenientes de las ciencias del hombre. Es decir, junto con los saberes enseñables en la escuela (biología, matemáticas, geografía e historia, por ejemplo), la formación de profesores incluyó, también, conocimientos procedentes de disciplinas como la sociología, la antropología y la psicología, lo cual implica reconocer que estamos en el marco de particulares formas de razonamiento sobre lo social. Importa, entonces, discutir los principios que genera el lenguaje de la pedagogía, entender la gramática que produce la comunicación pedagógica. En pocas palabras, la manera como el campo opera. En esta perspectiva, la pedagogía no es solo transmisión, por lo tanto, es necesario investigar la constitución de la comunicación pedagógica en sí.

Es preciso decir, para finalizar, que este trabajo investigativo sobre las páginas de estas dos revistas tituladas Educación, que circularon en São Paulo y Bogotá, entre 1932 y 1939, posibilitó reconocer que el discurso que circuló proveniente, por ejemplo, de la moderna psicología, contribuyó a configurar ciertas disposiciones en los profesores a través de las cuales se asumió la infancia y la manera en que, desde ese mirar, se actuó sobre el mundo. Disposiciones a través de las cuales los individuos más nuevos en el mundo, los recién llegados, los más pequeños, pudieron, en adelante, ser vistos como parte de un sistema de relaciones sociales: como niños escolarizados, educandos, en proceso de desarrollo, susceptibles de ser comparados y medidos. 
En este marco, reconocer la íntima vinculación del conocimiento experto y especializado con el ejercicio del poder, lleva a la pregunta de cómo pensar el conocimiento, considerado como práctica política, en la importante tarea de desnaturalizar, es decir, desfamiliarizar lo familiar, siguiendo la premisa de que lo que en cualquier momento dado se considera como conocimiento, se halla incluido en los conflictos sobre quién puede hablar y según qué criterios de verdad. ${ }^{30}$ La preocupación por el conocimiento, entonces, como un campo de práctica cultural, radica en entender los discursos que circularon en estas revistas como discursos que fueron puestos a disposición de los profesores y contribuyeron a contornear las prácticas pedagógicas. Al ser estos discursos un objeto de escrutinio, es posible evidenciar cómo cambian esas reglas con el transcurso del tiempo. La clasificación del conocimiento y la gestión de lo simbólico, así como sus usos y apropiaciones son fundamentales en esta pesquisa, al ser considerados como prácticas políticas.

Discutir la puntual lógica que presenta el campo de producción simbólica implica discutir "qué es hacer ciencia, o más precisamente, tratar de saber qué hace el científico, [ya que] no es solo interrogarse sobre la eficacia y el rigor formal de las teorías y de los métodos, es examinar a las teorías y [a] los métodos en su aplicación, para determinar qué hacen con los objetos y qué objetos hacen". ${ }^{31}$ Antes que como un resultado directo de la existencia de una realidad dada, las Revistas Impresas permiten comprender las cambiantes divisiones de verdad/falsedad, en la particularidad de cada uno de los contextos culturales donde fueron puestas en circulación. Desde esta perspectiva analítica, comprendida como un análisis del presente, se considera la razón como un campo de prácticas culturales que buscan ordenar las formas de definir los problemas y de comprender las posibilidades que para su solución fueron discutidas en diferentes periodos y para particulares contextos.

Es posible decir que mediante la perspectiva difundida desde estas dos revistas se buscó re-organizar el conocimiento sobre la práctica del docente, sobre los conocimientos a enseñar, sobre la individualidad del niño; en términos generales, sobre asuntos relacionados con el proceso educativo: aprendizaje, enseñanza, conocimiento, sujeto, contexto, lenguaje y comunicación, por ejemplo. Desde estas dos revistas impresas se vislumbra la pretensión, en cada uno de los artículos, por construir una perspectiva para comprender

30 Zemelman, 2005.

31 Bourdieu, Chamboredon y Passerón, (1973), 29. 
las condiciones sociales, culturales y políticas en estos dos contextos culturales. Como herramienta analítica, el concepto de discurso permite ir más allá de los dualismos que separan lo ideal de lo real, lo simbólico de lo material en la producción del significado. Desde esta perspectiva, el lenguaje y el discurso no son considerados como un reflejo de la realidad social, sino como constituyentes de esta. Se entiende que es a través del lenguaje y del discurso que la realidad social y psíquica inevitablemente se construye. El concepto de discurso posibilita indagar los procesos de formación de profesores, ya que continúa siendo un campo de combate y, por tanto, asunto por explorar.

\section{FUENTES}

Archivo del Observatorio Astronómico de Bogotá, Colombia.

Archivo del Gimnasio Moderno, Bogotá, Colombia.

Archivo Distrital de Bogotá, Colombia.

Archivo Pedagógico IDEP, Bogotá, Colombia

Biblioteca de la Universidad Pedagógica Nacional Bogotá, Colombia.

Biblioteca Nacional Bogotá, Colombia.

Biblioteca FE-UNICAMP, Campinas, Brasil.

Biblioteca FE-USP, São Paulo, Brasil.

Centro de Ciências, Letras e Artes, Campinas, Brasil.

Hemeroteca Luis López de Mesa: Biblioteca Luis Ángel Arango.

Museu Campos Salles, Campinas, Brasil.

Museu Carlos Gómes, Campinas, Brasil.

Revista Educação, São Paulo -1932 - 1939

Revista Educación, Bogotá -1933 - 1935

\section{REFERENCIAS}

Ariès, Philippe. História social da criança e da familia. Brasil: Editora Guanabara, 1978.

Almeida F., Ana Maria e Alice Nogueira. A escolarizaçao das elites. Um panorama internacional de pesquisa. Rio de Janeiro: Editora Vozes, Petrópolis, 2002.

Bachelard, Gastón. La formación del espíritu científico (1948). México: Siglo XXI Editores, 1973. 
Bortoleto Nery, Ana Clara. “Embates no campo educacional: a sociedade de educação de São Paulo (1922-1931)". UFSCar: Mimeo, s/f.

Bourdieu, Pierre y Jean-Claude Passeron. Los Herederos. Los estudiantes y la cultura (1964). Argentina: Siglo veintiuno editores, 2003.

Bourdieu, Pierre. Lección sobre la lección (1982). Barcelona: Anagrama, 2002.

Bourdieu, Pierre. El oficio de cientifico. Ciencia de la ciencia y reflexividad (2000). Barcelona: Anagrama, 2003.

Bourdieu, Pierre. El oficio de sociólogo (1973). México: Siglo XXI, 1994.

Bernstein, Basil. La construcción social del discurso pedagógico. Bogotá: Prodic, El Griot, 1990.

Bernstein, Basil. La estructura del discurso pedagógico. Madrid: Morata, 1990.

Bernstein, Basil. Pedagogía, control simbólico e identidad. Madrid: Morata, 1996.

Burke, Peter. Historia social del conocimiento. De Gutemberg a Diderot. Barcelona: Paidós, 2002.

Bustamante, Guillermo. Sujeto, sentido y formación. La educación vista desde el psicoanálisis, con sesgo lingüístico. Tesis de Doctorado en Educación, Doctorado Interinstitucional en Educación -DIE- en Universidad Pedagógica Nacional, 2011.

Claparède, E. L'École sur mesure. Genève: Payot, 1920.

Calvino, Ítalo. Palomar. Madrid: Alianza três, 1983.

Catani, Denice Barbara y Maria Helena Camara Bastos, (Org). Educação em Revista. A imprensa periódica e a Historia da Educação. São Paulo: Escrituras, 1997.

Chagas de Carvalho, Marta Maria. “Manuales de pedagogía, materialidad de lo impreso y circulación de modelos pedagógicos en el Brasil". Revista Colombiana de Educación, 52.(1997): 92-113.

Chagas de Carvalho, Marta Maria. Molde nacional y fôrma cívica. Universidade de São Francisco - CDAPH-IFAN: Campus de Bragança Paulista, 1998.

Chagas de Carvalho, Marta Maria. “O território do consenso e a demarcação do perigo: política e memória no debate educacional dos anos 30". En Memória intelectual da educação brasileira, organizado por Marcos Freitas. Brasil: Universidade São Francisco, CDAPH, 2002.

De Souzas Biccas, Maurilane. O impresso como estrategia de formação. Revista do ensino de Minas Gerais (1925-1940). Belo Horizonte: Argvmentvm, 2008.

Eco, Humberto. "A propósito de la "superioridad" occidental". Revista Foro Núo. 43 (2002): 4-8.

De Albuquerque Favero, Maria de Lourdes e Jader de Medeiros Brito. Diccionario de Educadores do Brasil. Da colónia aos dias atuais. Brasil: Editora UFRJ-MEC-INEP, 2002.

Desrosières, Alain. “¿Cómo fabricar cosas que se sostienen entre sí? Las ciencias sociales, la estadística y el Estado”. Archipiélago, N0. 20 (1995),19-31.

Díaz-Soler, Carlos Jilmar. A escola sob medida: Psicologia e Práticas Pedagógicas nas Revistas "Educação", em São Paulo e Bogotá (1932-1939), Tesis de Doctorado en Educación, Universidade Estadual de Campinas, UNICAMP, 2011, Brasil.

Elias, Norbert. La sociedad de los individuos. Barcelona: Península, 2011. 
Elias, Norbert. Conocimiento y poder. Madrid: Las ediciones de la Piqueta, 1994.

Ferrière, Adolphe. L'école sur mesure à la mesure du maître. Ginebra: Ateliers Atarkk, 1931.

Foucault, Michel. “A psicología de 1850 a 1950". En Problematição do sujeito, psicología, Psiquiatría e psicanalise, Brasil: Forense Universitaria, 2004.

Martha Cecilia Herrera y Carlos Low, Los intelectuales y el despertar cultural del siglo. El caso de la Escuela Normal Superior. Una historia reciente y olvidada. Universidad Pedagógica Nacional, 1994.

Nunes, Clarice. “O Estado novo e o debate educacional nos anos 30", en Memória intelectual da educação brasilera,, Em Marcos Cezar De Freitas. Brasil: Universidade São Francisco, CDAPH, 2002.

Prieto, Víctor Manuel. El Gimnasio Moderno y la formación de la Elite Liberal Bogotana. Bogotá: Universidad Pedagógica Nacional, 2000.

Popkewitz, Thomas. "La producción de la razón y el poder: historia del currículo y tradiciones intelectuales", en Rostros históricos de la educación. Miradas, estilos, recuerdos, Coordinado por María Esther Aguirre Lora. México: CESU - UNAM, Fondo de Cultura Económica, 2001.

Popkewitz, Thomas, Franklin Barry y Miguel Pereyra (Comp.). Historia cultural y educación. Ensayos críticos sobre conocimiento y educación. Barcelona: Ediciones Pomares, 2003.

Quiceno, Humberto, Javier Sáenz Obregón y Luis Arturo Vahos. “La instrucción y la educación pública en Colombia: 1903-1997", en. Modernización de los sistemas educativos iberoamericanos, Siglo XX, Tomo II. Compilado por, Olga Lucia Zuluaga Garcés y Gabriela Ossembach Sauter. Bogotá: Cooperativa Editorial Magisterio, Colciencias-Universidad de Antioquia, 2004.

Sáenz, Javier, Oscar Saldarraiga y Armando OSPINA. Mirar la infancia: pedagogía, moral y modernidad en Colombia, 1903-1956. Colombia: Conciencias, Foro Nacional por Colombia, Universidad de Antioquia, 1997.

Schwartzman, Simon, Helena Maria Busquet Bomeny, Vanda Maria Ribeiro Costa. Tempos de Capanema. Sao Paulo: EDUSP-Paz e Terra, 1984.

Serna Dímas, Adrián, "Saberes educativos y saberes sociales: esbozo para una historia social de sus estructuras sociales", en Estructura tiempo y sujeto. Universidad Distrital Francisco José de Caldas (2006).

Spedo Hilsdorf, Jorge Warde, Chagas de Carvalho, "Apontamentos sobre a história da escola e do sistema escolar no Brasil", en Génesis y desarrollo de los Sistemas Educativos Iberoamericanos Siglo XIX, 2004.

Wallerstein, Immanuel (coord.). Abrir las ciencias sociales. Reporte de la Comisión Gulbelkian para la reestructuración de las ciencias sociales. México: Siglo XXI, 1996.

Warde, Miriam Jorge. "Para uma história disciplinar: psicologia, criança e pedagogia". En História Social da Infância no Brasil. En Marcos Cezar De Freitas. São Paulo: Cortes Editora, 2006.

Xavier, Maria do Carmo (Org.). Manifesto dos pioneiros da educação. Um legado educacional em debate. Rio de Janeiro: FGV Editora, 2004. 
Zemelman, Hugo. Sujeto: Existencia y potencia. México: Anthropos, 1998.

Zemelman, Hugo. Conocimiento y ciencias sociales. Algunas lecciones sobre problemas epistemológicos. México: Universidad de la Ciudad de México 2005.
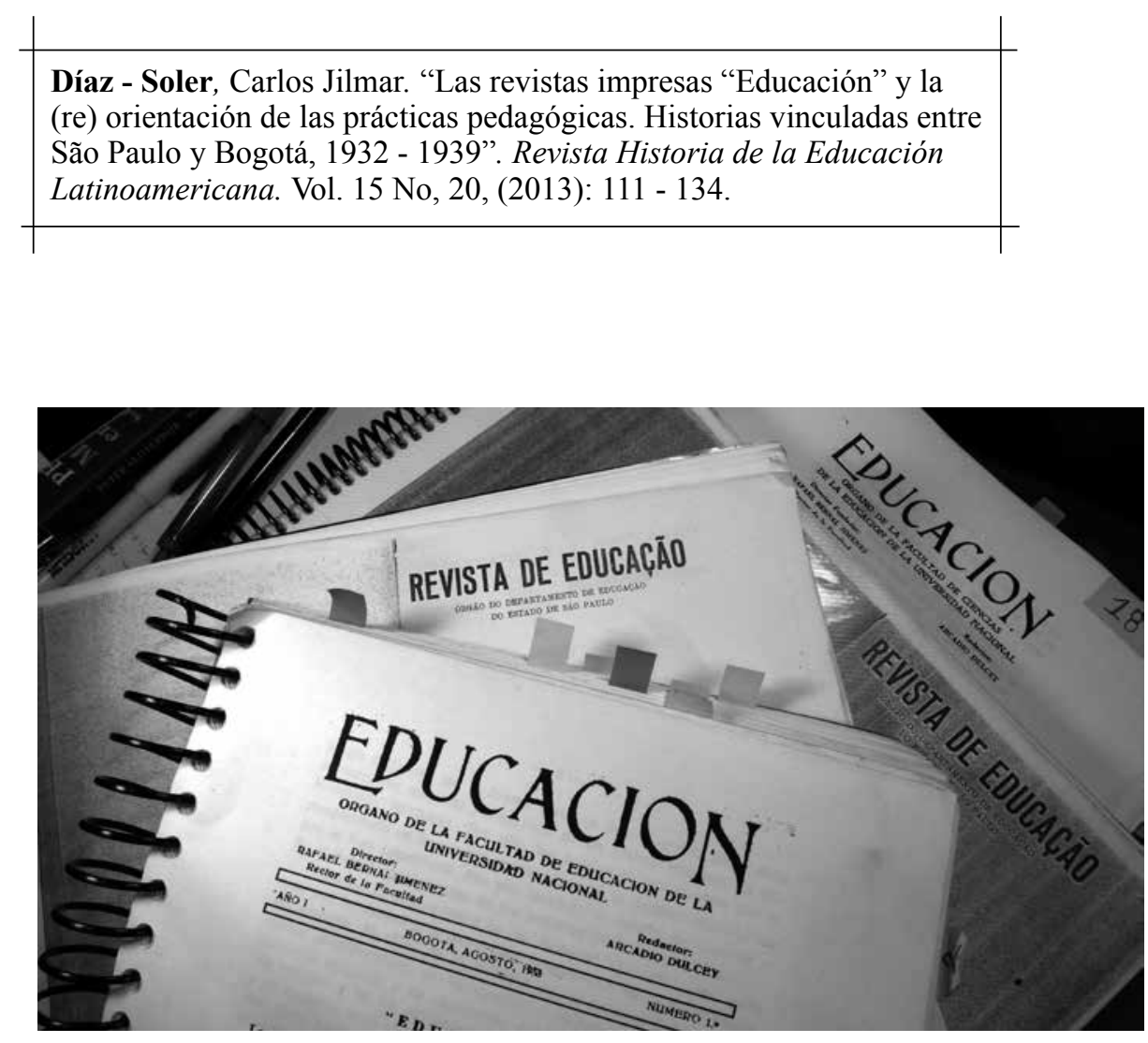